\title{
Hydration-Induced Structural Transitions in Biomimetic Tandem Repeat Proteins
}

\author{
Romeo C. A. Dubini ${ }^{1,2}$, Huihun Jung ${ }^{3}$, Chloe H. Skidmore ${ }^{3}$, Melik C. Demirel ${ }^{3,}{ }^{*}$, and Petra \\ Rovó $^{1,2, *}$ \\ ${ }^{1}$ Faculty of Chemistry and Pharmacy, Department of Chemistry, Ludwig-Maximilians-Universität München, 81377 \\ Munich, Germany \\ ${ }^{2}$ Center for Nanoscience (CeNS), Faculty of Physics, Ludwig-Maximilians-Universität München, Schellingstraße 4, \\ 80799 Munich, Germany \\ ${ }^{3}$ Center for Research on Advanced Fiber Technologies (CRAFT), Materials Research Institute, Department of \\ Engineering Science and Mechanics, and Huck Institutes of Life Sciences, Pennsylvania State University, University \\ Park, Pennsylvania 16802, United States of America \\ *Corresponding author: Melik C. Demirel (melik@psu.edu), Petra Rovó (petra.rovo@lmu.de)
}

\section{Table of Contents}

1 Supplementary Methods $\quad$ S2

1.1 Protein sequence . . . . . . . . . . . . . . . . . . . . . . S2

1.2 Hydrodynamic radius calculation . . . . . . . . . . . . . . . . . . . . S2

\section{List of Figures}

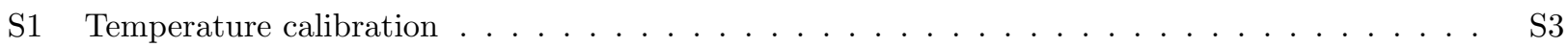

S2 Pulse sequence of the refocused INEPT-based $2 \mathrm{D}{ }^{13} \mathrm{C}-{ }^{13} \mathrm{C}$ experiment . . . . . . . . . . . . S3

S3 MALDI-TOF spectra of $\mathrm{TR}(1,11)$ protein . . . . . . . . . . . . . . . . . . S4

S4 FTIR spectra of $\mathrm{TR}(1,11)$ amide I and II regions . . . . . . . . . . . . . . . . . . . . . . S4

S5 Secondary structure assessment based on ${ }^{13} \mathrm{C}$ chemical shift difference . . . . . . . . . . . . . . S4

S6 $J(0), J\left(\omega_{\mathrm{N}}\right)$ and $J\left(0.87 \omega_{\mathrm{H}}\right)$ spectral density values . . . . . . . . . . . . . . . . S5

$\mathrm{S} 7 \quad 1 \mathrm{D}{ }^{13} \mathrm{C}$ spectra of $\mathrm{HF}, \mathrm{AF}$, and $\mathrm{AP} \ldots \ldots \ldots \ldots \ldots$

S8 Aliphatic region of the $1 \mathrm{D}{ }^{13} \mathrm{C}$ spectra of $\mathrm{HF} \ldots \ldots \ldots \ldots$

S9 $\quad{ }^{1} \mathrm{H}_{-}{ }^{15} \mathrm{~N}$ CP-based $2 \mathrm{D}$ spectra of $\mathrm{AP}, \mathrm{AF}$, and $\mathrm{HF} \ldots \ldots \ldots \ldots$

$\mathrm{S} 10{ }^{13} \mathrm{C} \alpha{ }^{-13} \mathrm{CO}$ region of the 2D DREAM spectra of sample AP, AF, and HF . . . . . . . . . . . S S

$\mathrm{S} 11{ }^{1} \mathrm{H}_{-}{ }^{13} \mathrm{CO} \mathrm{CP}$-based $2 \mathrm{D}$ spectra of sample AP, AF, and $\mathrm{HF} \ldots \ldots \ldots$. . . . . . . . . . . S8

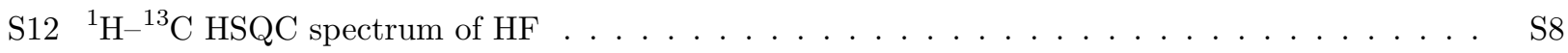

S13 Comparison of the methyl region of the ${ }^{1} \mathrm{H}^{-}{ }^{13} \mathrm{C}$ HSQC spectra of AP, AF, and HF . . . . . . . S9

\section{List of Tables}

S1 Deconvoluted FTIR spectral data for cast and washed films. . . . . . . . . . . . . . . . . . . S10

$\mathrm{S} 2 \quad{ }^{13} \mathrm{C}$ chemical shift of $\mathrm{TR}(1,11)$ compared to literature values . . . . . . . . . . . . . . . S10 


\section{Supplementary Methods}

\subsection{Protein sequence}

MGTLSYGYGGLYGGLYGGLGYGPAAASVSTVHHP

STGTLSYGYGGLYGGLYGGLGYGPAAASVSTVHHP

STGTLSYGYGGLYGGLYGGLGYGPAAASVSTVHHP

STGTLSYGYGGLYGGLYGGLGYGPAAASVSTVHHP

STGTLSYGYGGLYGGLYGGLGYGPAAASVSTVHHP

STGTLSYGYGGLYGGLYGGLGYGPAAASVSTVHHP

STGTLSYGYGGLYGGLYGGLGYGPAAASVSTVHHP

STGTLSYGYGGLYGGLYGGLGYGPAAASVSTVHHP

STGTLSYGYGGLYGGLYGGLGYGPAAASVSTVHHP

STGTLSYGYGGLYGGLYGGLGYGPAAASVSTVHHP

STGTLSYGYGGLYGGLYGGLGYGPAAASVSTVHHP

STGTLSYGYGGLYGGLYGGLGYGP

Black, red and blue segments indicate the cleavage site, the amorphous and the crystalline region, respectively.

\subsection{Hydrodynamic radius calculation}

For a large solute molecule of radius $R$ in a solvent of much smaller molecules, the diffusion coefficient $D$ of the solute is described via the Stokes-Einstein relationship:

$$
R_{h}=\frac{k_{B} T}{\pi D \eta \epsilon}
$$

where $R_{h}$ is the hydrodynamic radius of the molecule, $k_{B}$ is the Boltzmann constant, $T$ is the temperature of the system, $\eta$ is the solvent viscosity and $\epsilon$ is a constant determined by the choice of stick $(\epsilon=6)$ or slip $(\epsilon=4)$ hydrodynamic boundary conditions at the solute surface. Consistently with previous investigations on polymers of similar molecular mass, we opted for constant $\epsilon=4$ (ref. [1]) which resulted in $R_{h}=33.0 \AA$.

To estimate the hydrodynamic radius of a folded $\left(R_{h}^{\text {folded }}\right)$, denatured $\left(R_{h}^{\text {denatured }}\right)$, or intrinsically disordered protein $\left(R_{h}^{\mathrm{IDP}}\right)$ which has an equal number of residues as $\operatorname{TR}(1,11)$, i.e. $N=404$, we used a reported power-low relationship:

$$
\begin{gathered}
R_{h}^{\text {folded }}=4.92 N^{0.285} \\
R_{h}^{\text {denatured }}=2.33 N^{0.549} \\
R_{h}^{\mathrm{IDP}}=2.49 N^{0.509}
\end{gathered}
$$

The above empirical relations have been derived from pulse-field gradient NMR measurements performed in aqueous solutions.[2] Protein solvation by DMSO can affect the hydrodynamic radius, especially the extent of the solvation shell around the molecule. 

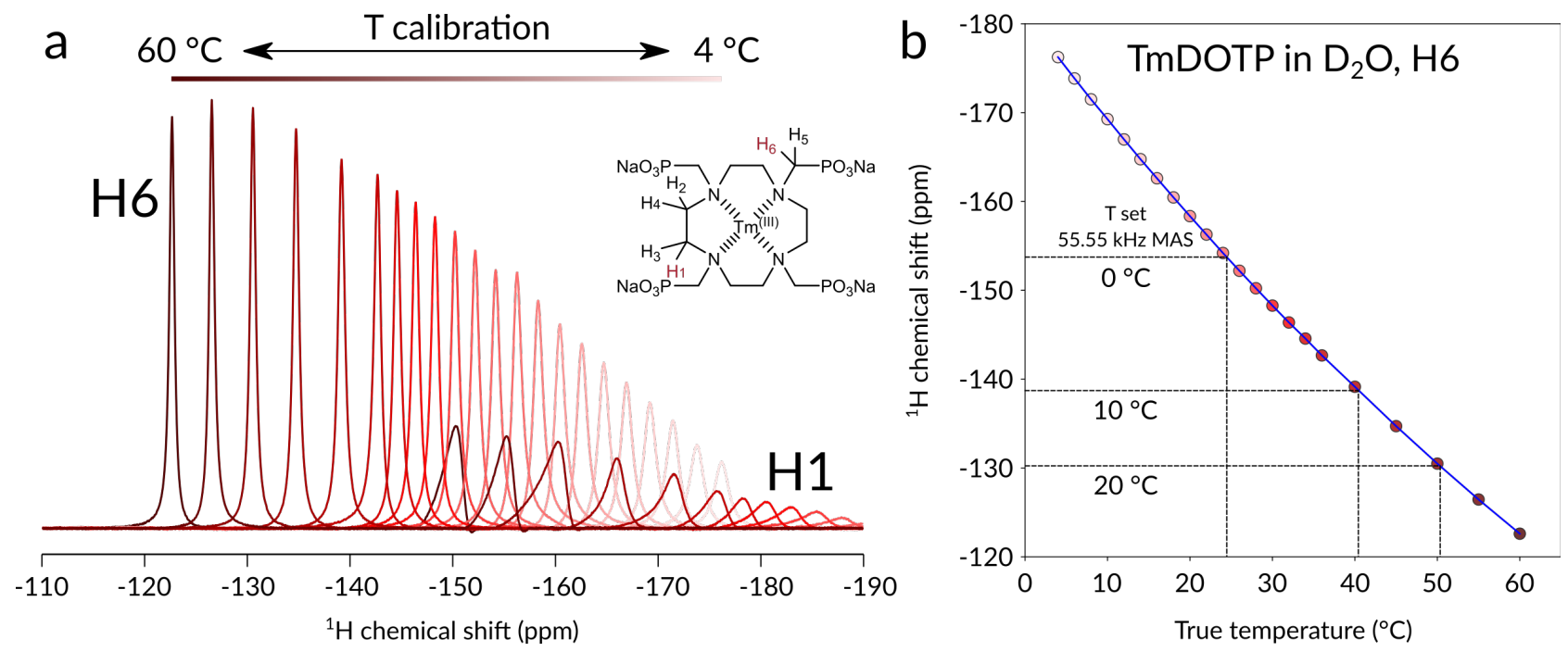

Figure S1: (a) Solution-state TmDOTP temperature calibration via $1 \mathrm{D}^{1} \mathrm{H}$ NMR. Proton H6 was measured inbetween 4 and $60{ }^{\circ} \mathrm{C}$. (b) The solution-state calibration data was used to determine the true temperature of a MAS solid-state $\mathrm{TR}(1,11)$ sample spinning at $55.55 \mathrm{kHz}$.

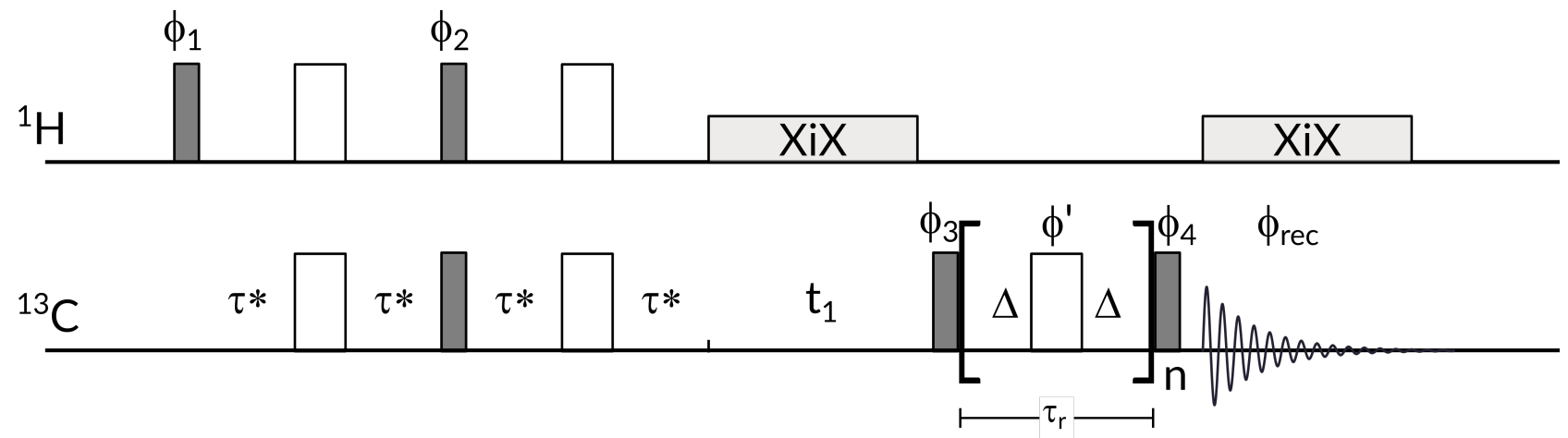

Figure S2: Pulse sequence of the refocused INEPT-based $2 \mathrm{D}{ }^{13} \mathrm{C}-{ }^{13} \mathrm{C}$ experiment using RFDR mixing for magnetization transfer. The initial ${ }^{13} \mathrm{C}$ polarization is created via a refocused INEPT step where $\tau^{*} \approx 0.45(1 / 4 J)$ in order to mitigate excessive coherence decay during the refocused INEPT step. ${ }^{3}$ Following the indirect evolution period $t_{1}$, a non-selective $\pi / 2$ generates longitudinal ${ }^{13} \mathrm{C}$ magnetization and rotor-synchronized $\pi$ pulses are applied once per rotor period $\left(\tau_{r}\right)$ for $n=256$ times. $\pi$ pulses are phase-cycled according to the XY-16 scheme in order to minimize the pulse imperfections. An XiX decoupling is applied during the indirect and direct acquisition time. Pulse phases are $\phi_{1}=x,-x, \phi_{2}=2(-y), 2(y), \phi_{3}=4(y), 4(-y), \phi_{\text {rec }}=x,-x,-x, x,-x, x, x,-x$; $\phi_{4}$ is decremented by $90^{\circ}$ to achieve quadrature detection of $t_{1}$ according to the States-TPPI scheme. 


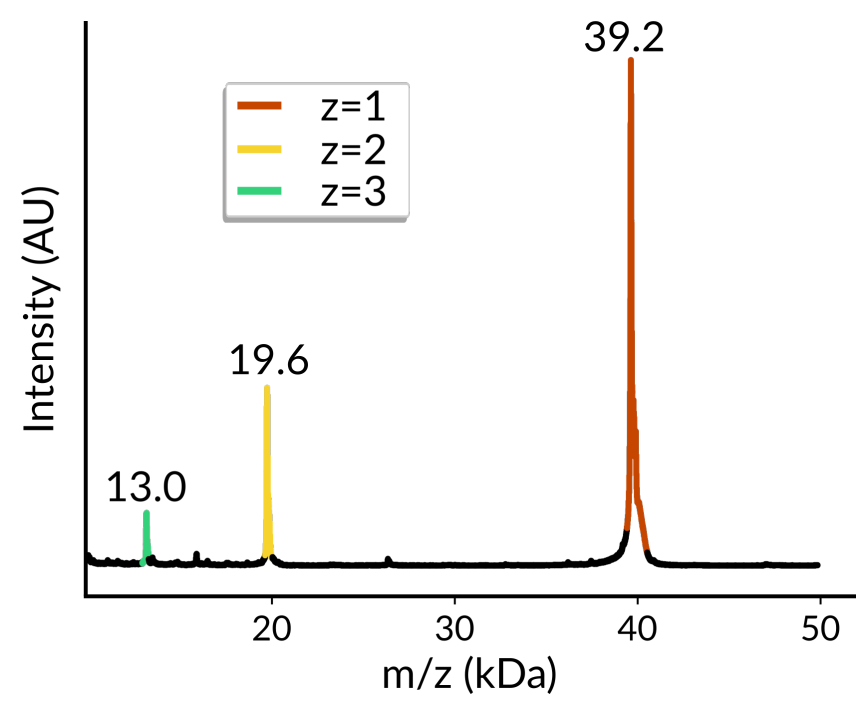

Figure S3: MALDI-TOF spectra of TR $(1,11)$ protein. Peaks are labeled by color according to the appropriate mass-to-charge ratio.

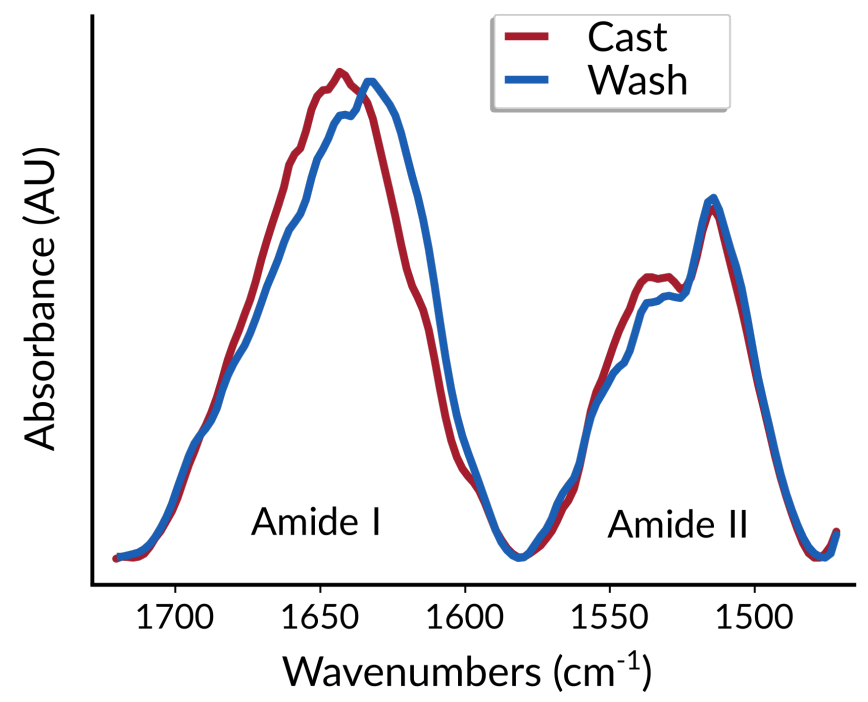

Figure S4: FTIR spectra of TR(1,11) amide I and II regions.

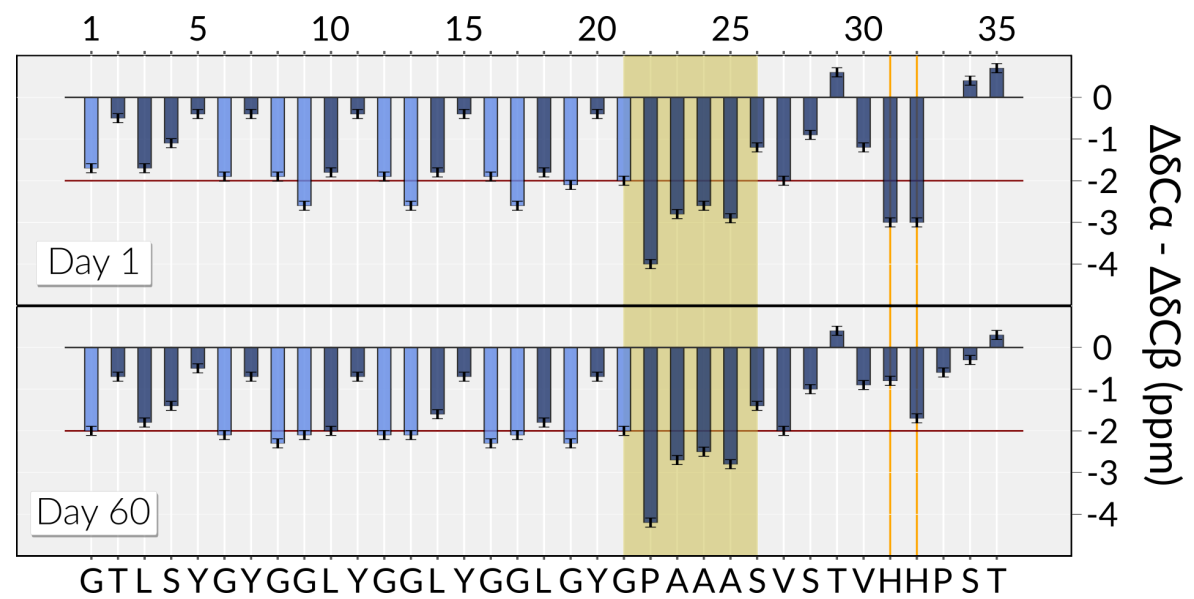

Figure $\mathrm{S} 5: \Delta \delta \mathrm{C} \alpha-\Delta \delta \mathrm{C} \beta$ chemical shifts reflect structuring propensities in $\mathrm{TR}(1,11)$ where $\Delta \delta \mathrm{C} i=\delta \mathrm{C}_{\mathrm{obs}}-\delta \mathrm{C}_{\mathrm{rc}}$ is defined as the deviation between the observed "obs") and the random coil ("rc") chemical shifts of the $i^{\text {th }}$ carbon. Gly $\Delta \delta \mathrm{C} \alpha$ values are displayed in light blue. Yellow shade indicates a segment with $\beta$-sheet structural preference. Vertical orange lines mark the sites which change conformation during the 60 days of incubation. 

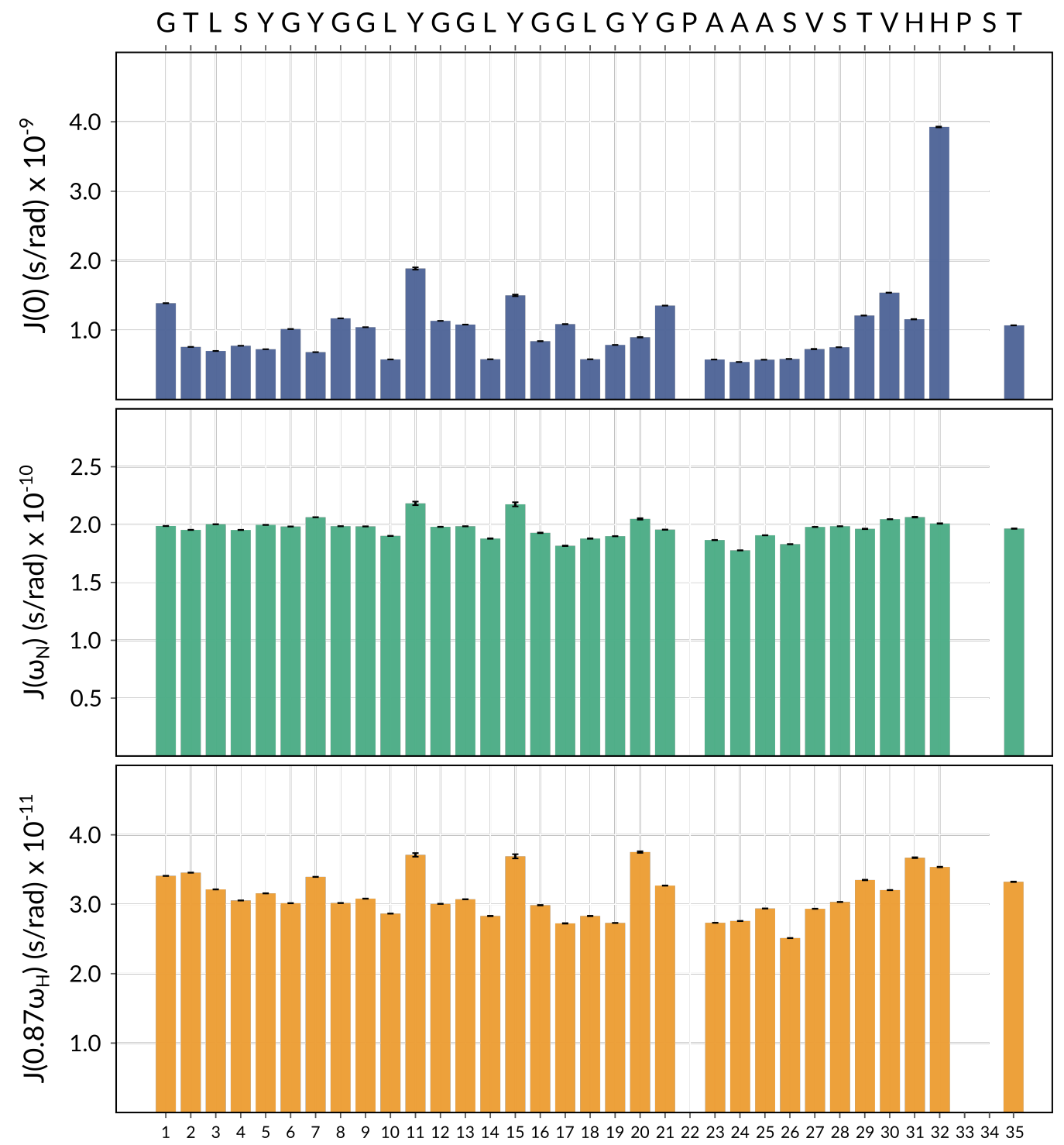

Figure S6: $J(0), J\left(\omega_{\mathrm{N}}\right)$ and $J\left(0.87 \omega_{\mathrm{H}}\right)$ spectral density values derived from the ${ }^{15} \mathrm{~N}$ relaxation rates and heteronuclear nOe experiments measured at $800 \mathrm{MHz}{ }^{1} \mathrm{H}$ Larmor frequency at $37{ }^{\circ} \mathrm{C}$. 


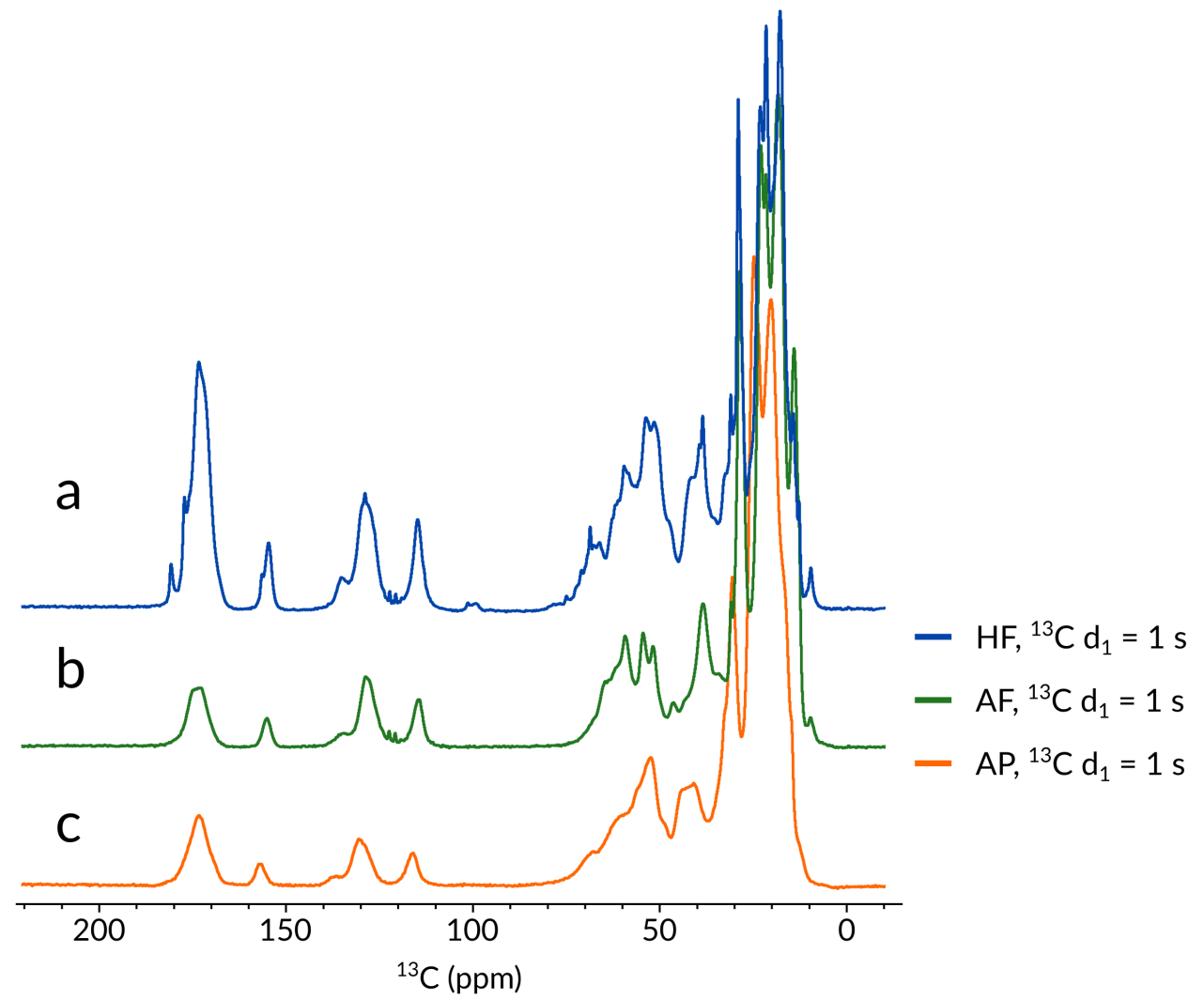

Figure S7: Comparison of the $1 \mathrm{D}{ }^{13} \mathrm{C}$ direct excitation spectra of the $\mathrm{HF}$ (a), AF (b), and AP (c) samples recorded at $700 \mathrm{MHz}{ }^{1} \mathrm{H}$ Larmor frequency, at $55.55 \mathrm{kHz}$ MAS, at $25{ }^{\circ} \mathrm{C}$ nominal temperature. All spectra were recorded for 8192 scans and a recycle delay of $1 \mathrm{~s}$.

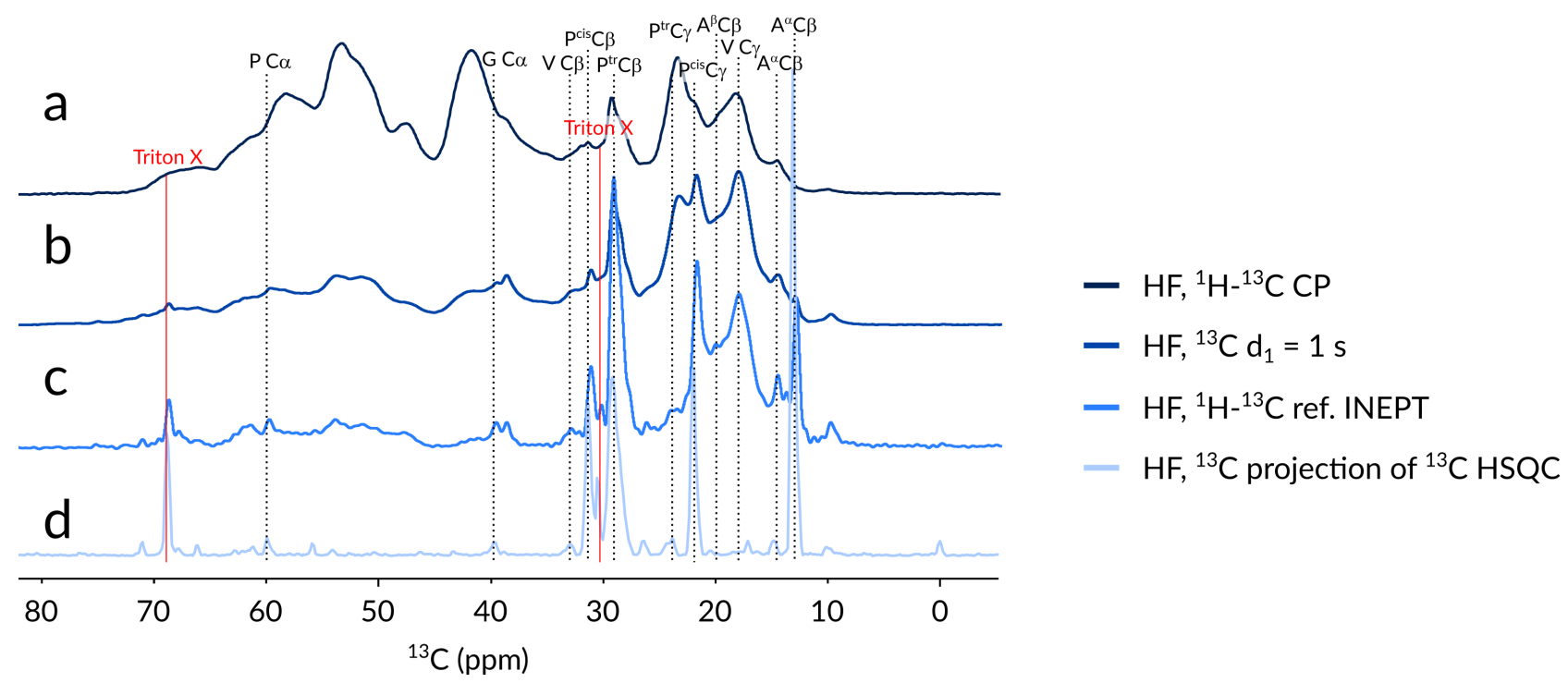

Figure S8: Comparison of the aliphatic region of the $1 \mathrm{D}{ }^{13} \mathrm{C}$ spectra of the $\mathrm{HF}$ sample recorded at $700 \mathrm{MHz}{ }^{1} \mathrm{H}$ Larmor frequency, at $55.55 \mathrm{kHz}$ MAS, at $25{ }^{\circ} \mathrm{C}$ nominal temperature. Tentative signal assignment is displayed on the top. Labels "Triton- $\mathrm{X}$ " indicate the position of the detergent signals. (a) ${ }^{1} \mathrm{H}-{ }^{13} \mathrm{C} \mathrm{CP}$ spectrum recorded with a $0.9 \mathrm{~ms}$ long zero-quantum $\mathrm{CP}\left(66 \mathrm{kHz}\right.$ tangential-shaped $\mathrm{CP}$ on ${ }^{1} \mathrm{H}$ and a $11 \mathrm{kHz}$ rectangular shaped $\mathrm{CP}$ on ${ }^{13} \mathrm{C}$ ) with carrier offset at $52 \mathrm{ppm}$; (b) Direct excitation ${ }^{13} \mathrm{C}$ spectrum with $13 \mathrm{kHz}$ XiX ${ }^{1} \mathrm{H}$-decoupling during acquisition; (c) ${ }^{1} \mathrm{H}^{-13} \mathrm{C}$ refocused INEPT spectrum recorded with an INEPT transfer of $800 \mu \mathrm{s}$; (d) ${ }^{13} \mathrm{C}$ projection of the $2 \mathrm{D}{ }^{1} \mathrm{H}_{-}{ }^{13} \mathrm{C}$ HSQC spectrum recorded with an INEPT transfer time of $2000 \mu$ s. Carbons with high mobility on the ps-ns time scale have disproportionally higher signal intensities in spectrum (b), (c), and (d). 


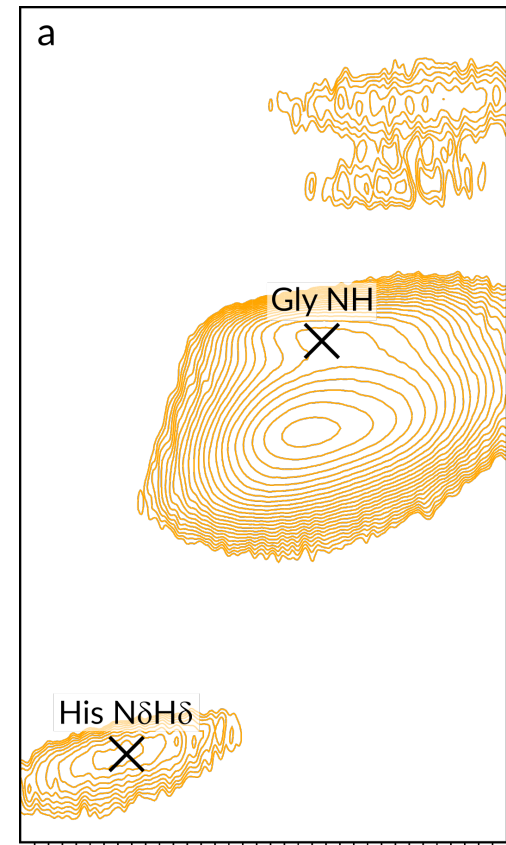

$\begin{array}{lllllll}12 & 11 & 10 & 9 & 8 & 7\end{array}$
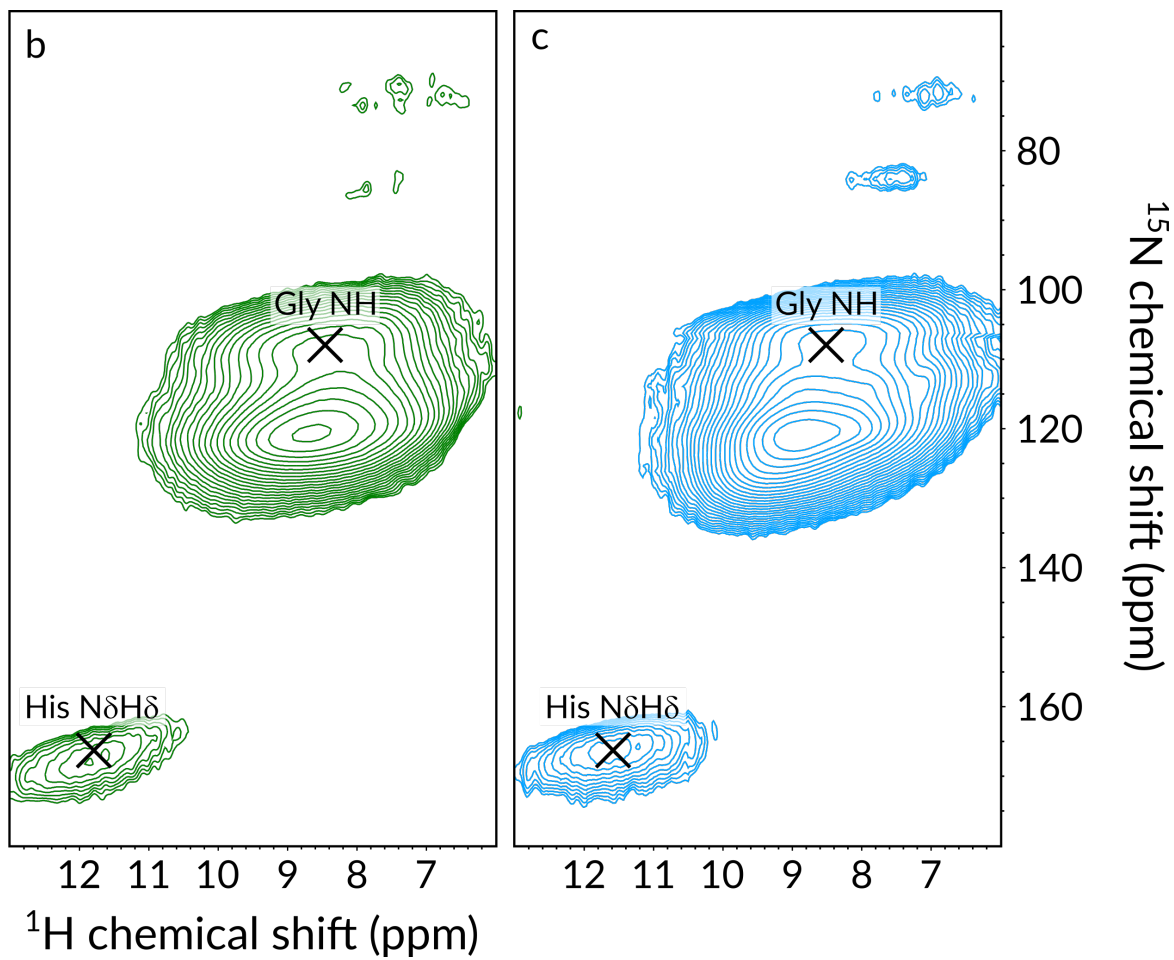

$\mathrm{His} \mathrm{N} \delta \mathrm{H} \delta$

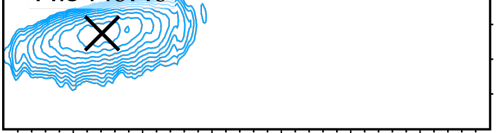

160

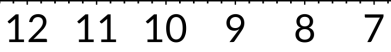

Figure S9: ${ }^{1} \mathrm{H}-{ }^{15} \mathrm{~N}$ CP-based 2D spectra of sample AP (a), AF (b) and $\mathrm{HF}(\mathrm{c})$ of ${ }^{13} \mathrm{C},{ }^{15} \mathrm{~N}$-labeled $\mathrm{TR}(1,11)$ recorded at $700 \mathrm{MHz}{ }^{1} \mathrm{H}$ Larmor frequency at $55.55 \mathrm{kHz}$ MAS at $25{ }^{\circ} \mathrm{C}$.
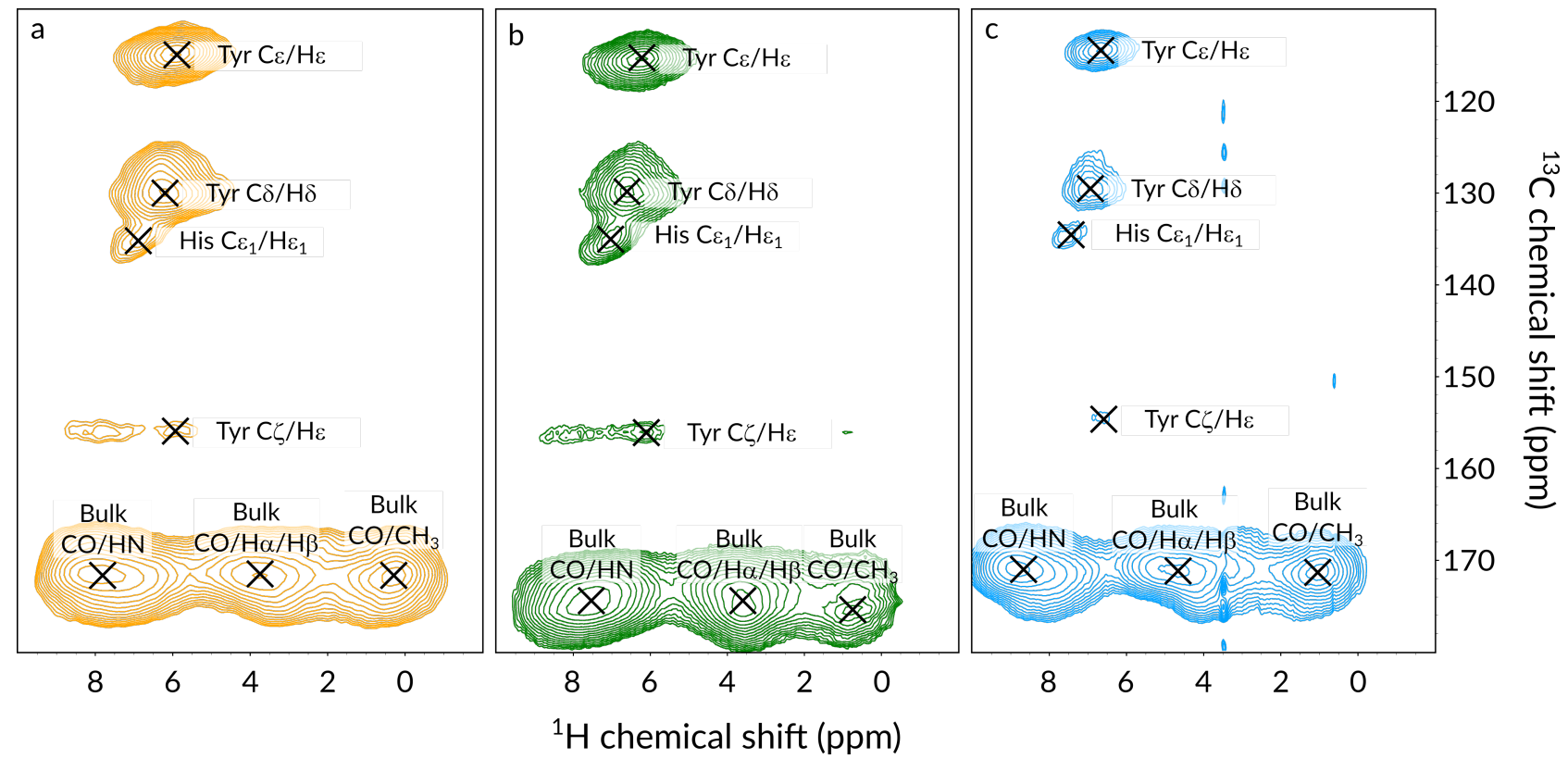

Figure S10: ${ }^{1} \mathrm{H}^{13} \mathrm{CO}$ CP-based 2D spectra of sample AP (a), AF (b), and HF (c) of ${ }^{13} \mathrm{C},{ }^{15} \mathrm{~N}$-labeled $\mathrm{TR}(1,11)$ recorded at $700 \mathrm{MHz}{ }^{1} \mathrm{H}$ Larmor frequency at $55.55 \mathrm{kHz}$ MAS at $25{ }^{\circ} \mathrm{C}$. 


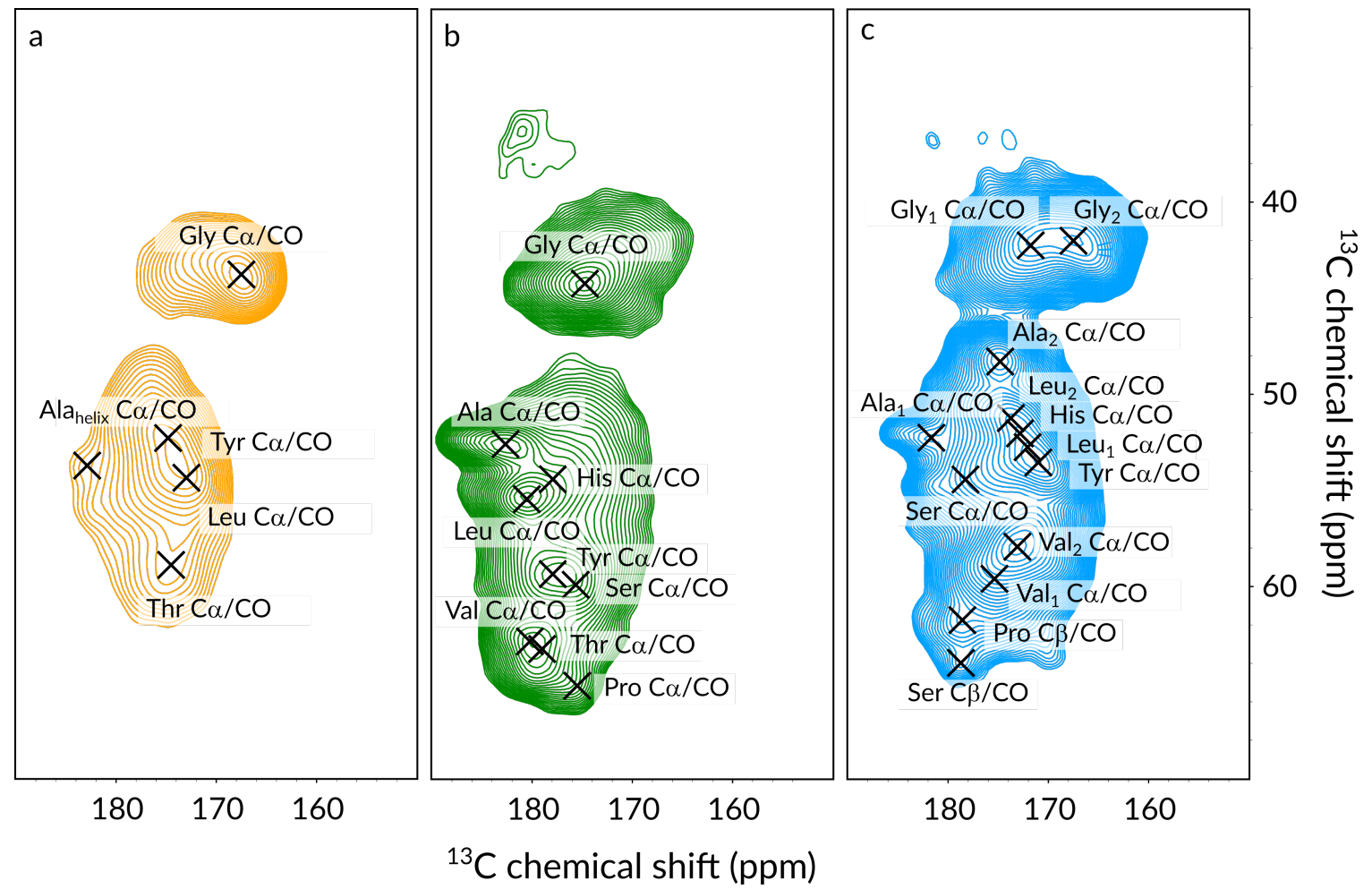

Figure S11: ${ }^{13} \mathrm{C} \alpha-{ }^{13} \mathrm{CO}$ CP-based 2D spectra of sample AP (a), AF (b), and $\mathrm{HF}(\mathrm{c})$ of ${ }^{13} \mathrm{C},{ }^{15} \mathrm{~N}$-labeled TR $(1,11)$ recorded at $700 \mathrm{MHz}{ }^{1} \mathrm{H}$ Larmor frequency at $55.55 \mathrm{kHz}$ MAS at $25{ }^{\circ} \mathrm{C}$.

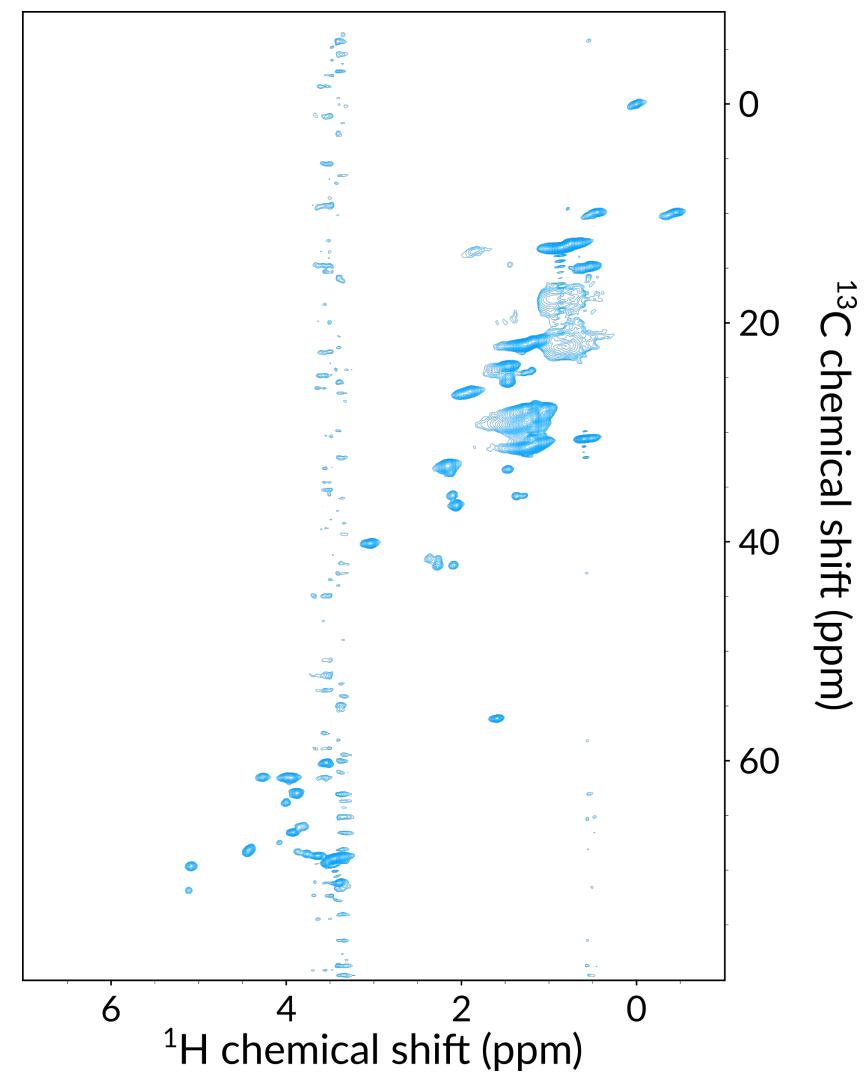

Figure S12: ${ }^{1} \mathrm{H}_{-}{ }^{13} \mathrm{C}$ HSQC spectrum of sample $\mathrm{HF}$ of ${ }^{13} \mathrm{C},{ }^{15} \mathrm{~N}$-labeled $\mathrm{TR}(1,11)$ recorded at $700 \mathrm{MHz}{ }^{1} \mathrm{H}$ Larmor frequency at $55.55 \mathrm{kHz}$ MAS at $55^{\circ} \mathrm{C}$. 


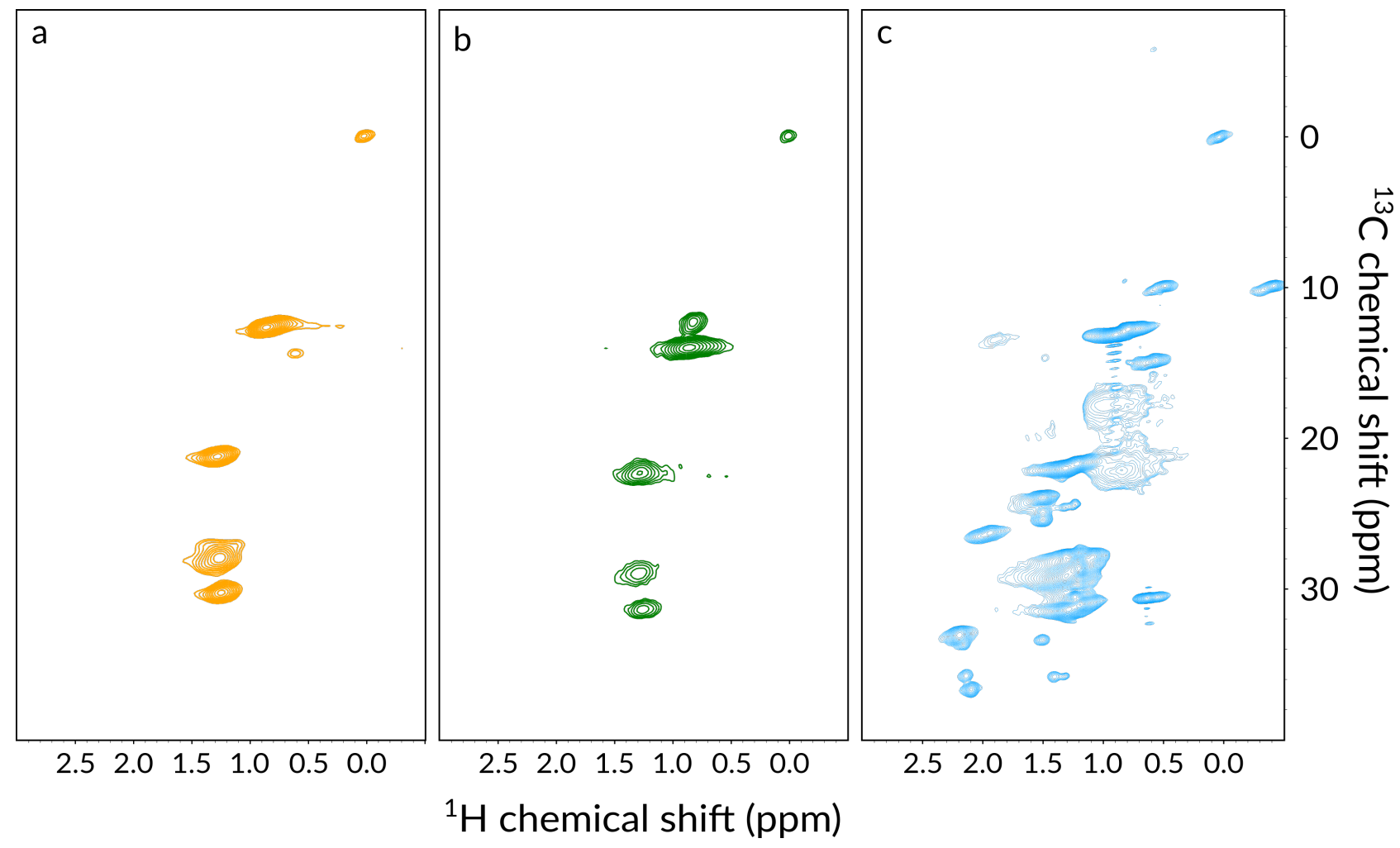

Figure S13: Comparison of the methyl region of the ${ }^{1} \mathrm{H}^{13}{ }^{13} \mathrm{C}$ HSQC spectrum of sample AP (a), AF (b), and $\mathrm{HF}(\mathrm{c})$ of ${ }^{13} \mathrm{C}$, ${ }^{15} \mathrm{~N}$-labeled $\mathrm{TR}(1,11)$ recorded at $700 \mathrm{MHz}{ }^{1} \mathrm{H}$ Larmor frequency at $55.55 \mathrm{kHz}$ MAS; the nominal temperature of (a) and (b) was $25^{\circ} \mathrm{C}$, and that of (c) was $55^{\circ} \mathrm{C}$. 
Table S1: Deconvoluted FTIR spectral data for cast and washed films.

\begin{tabular}{cccc} 
Assignment & Band position $\left(\mathbf{c m}^{-1}\right)$ & AF (\%) & HF (\%) \\
\hline$\alpha$-helix & $1658-1662$ & 25.3 & 7.1 \\
$\beta$-sheet & $1630-1632 / 1697-1703$ & 26.2 & 46.8 \\
Turn & $1665-1693$ & 14.0 & 22.2 \\
Side-chain & $1594-1597$ & 3.6 & 4.7 \\
Random Coil & $1640-1655$ & 30.9 & 19.3 \\
\hline
\end{tabular}

Table S2: ${ }^{13} \mathrm{C}$ chemical shift of TR $(1,11)$ compared to literature values. The random coil, $\beta$-strand and $\alpha$-helix chemical shifts (labeled as "PACSY database") are reported as the statistical average for 3000+ folded proteins. Reference values are also reported for spider ( N. clavipes) and silkworm (B. mori) silks ("Natural silks"). For all Pro entries, values for the trans isomer were reported. All shifts were directly referenced to DSS unless labeled with the ${ }^{*}$ symbol, were conversion from TMS was achieved via addition of 1.7 ppm. Symbols "-" and "/" indicate a chemical shift range or multiple distinct environments, respectively.

\begin{tabular}{|c|c|c|c|c|c|c|c|c|c|}
\hline \multirow[b]{2}{*}{ Residue } & \multirow[b]{2}{*}{ Index } & \multicolumn{3}{|c|}{ PACSY database ${ }^{4}$} & \multicolumn{2}{|c|}{ As spun silks } & \multicolumn{3}{|c|}{ Experimental TR(1,11) values } \\
\hline & & Coil & $\beta$-strand & $\alpha$-helix & Dragline ${ }^{* 5,6}$ & B. Mori $^{* 7}$ & DMSO- $d_{6}$ & $\mathbf{A F}$ & HF \\
\hline Gly $\mathrm{H}^{\mathrm{N}}$ & \multirow{5}{*}{1} & 8.34 & 8.35 & 8.28 & - & - & 8.06 & - & 8.4 \\
\hline Gly $\mathrm{H}_{\alpha}$ & & 3.93 & - & - & - & - & $3.72-3.82$ & - & - \\
\hline Gly CO & & 173.9 & 172.1 & 175.4 & 172.7 & 171.0 & 170.5 & $170.0-172.3$ & $168.2 / 170.3$ \\
\hline Gly C $\alpha$ & & 45.4 & 45.1 & 46.9 & 45.0 & 44.2 & 43.4 & $42.2-45.0$ & 41.6 \\
\hline Gly $\mathrm{N}^{\mathrm{H}}$ & & 110.0 & 109.8 & 107.9 & - & - & 106.9 & - & 107.6 \\
\hline Thr $H^{\mathrm{N}}$ & \multirow{7}{*}{2} & 8.20 & 8.58 & 8.04 & - & - & $7.78 / 7.82$ & - & - \\
\hline Thr $\mathrm{H}_{\alpha}$ & & 4.46 & - & - & - & - & 4.29 & - & - \\
\hline Thr CO & & 174.5 & 173.6 & 176.0 & - & - & 171.2 & 174.4 & 171.1 \\
\hline Thr C $\alpha$ & & 61.4 & 61.0 & 65.5 & - & - & 59.4 & 63.2 & 58.3 \\
\hline $\operatorname{Thr} \mathrm{C} \beta$ & & 69.4 & 70.4 & 68.1 & - & - & 68.1 & 66.2 & $66.5-68.4$ \\
\hline $\operatorname{Thr} \mathrm{C} \gamma$ & & 21.6 & - & - & - & - & 20.8 & 18.0 & 18.3 \\
\hline Thr $\mathrm{N}^{\mathrm{H}}$ & & 114.3 & 117.6 & 114.7 & - & - & 111.0 & - & - \\
\hline Leu $\mathrm{H}^{\mathrm{N}}$ & \multirow{8}{*}{3} & 8.10 & 8.72 & 8.07 & - & - & 7.93 & - & - \\
\hline Leu $\mathrm{H}_{\alpha}$ & & 4.31 & - & - & - & - & $4.30-4.38$ & - & - \\
\hline Leu CO & & 176.4 & 175.4 & 178.2 & - & - & 173.5 & 175.5 & $170.7 / 171.5$ \\
\hline Leu C $\alpha$ & & 54.7 & 53.8 & 57.4 & - & - & 52.4 & 54.4 & $50.4 / 52.0$ \\
\hline Leu $\mathrm{C} \beta$ & & 42.3 & 44.1 & 41.4 & - & - & 41.9 & 38.3 & $38.8 / 42.5$ \\
\hline Leu $\mathrm{C} \gamma$ & & 26.9 & - & - & - & - & 25.3 & 23.1 & $23.3-23.7$ \\
\hline Leu C $\delta$ & & 24.5 & - & - & - & - & 24.3 & $18.4 / 21.4$ & 21.5 \\
\hline Leu $\mathrm{N}^{\mathrm{H}}$ & & 122.0 & 124.7 & 120.0 & - & - & 120.1 & - & - \\
\hline Ser $H^{N}$ & \multirow{6}{*}{4} & 8.28 & 8.55 & 8.14 & - & - & 7.88 & - & - \\
\hline Ser $\mathrm{H}_{\alpha}$ & & 4.48 & - & - & - & - & $4.29 / 4.36$ & - & - \\
\hline Ser CO & & 174.4 & 173.5 & 176.0 & - & 173.9 & 171.4 & 173.1 & 173.9 \\
\hline Ser $\mathrm{C} \alpha$ & & 58.2 & 57.1 & 61.0 & 57.2 & 56.3 & 56.3 & 59.0 & 53.7 \\
\hline Ser $\mathrm{C} \beta$ & & 63.5 & 64.8 & 62.5 & 63.3 & 65.6 & 63.0 & 59.3 & 62.8 \\
\hline Ser $\mathrm{N}^{\mathrm{H}}$ & & 116.3 & 117.4 & 115.1 & - & - & 113.3 & - & - \\
\hline Tyr $\mathrm{H}^{\mathrm{N}}$ & \multirow{4}{*}{5} & 8.05 & 8.77 & 8.10 & - & - & 7.91 & - & - \\
\hline Tyr $\mathrm{H}_{\alpha}$ & & 4.61 & - & - & - & - & $4.45-4.51$ & - & - \\
\hline Tyr CO & & 174.9 & 174.3 & 177.1 & - & - & 173.0 & 173.9 & 170.1 \\
\hline Tyr C $\alpha$ & & 57.4 & 56.6 & 60.6 & - & - & 55.8 & 58.4 & 53.0 \\
\hline
\end{tabular}




\begin{tabular}{|c|c|c|c|c|c|c|c|c|c|}
\hline Tyr C $\beta$ & \multirow{6}{*}{5} & 38.9 & 41.0 & 38.1 & - & - & 37.8 & 34.7 & 38.5 \\
\hline Tyr C $\gamma$ & & 123.7 & - & - & - & - & - & 126.5 & 126.3 \\
\hline Tyr C $\delta$ & & 133.1 & - & - & - & - & 131.6 & 129.0 & 129.6 \\
\hline Tyr C $\epsilon$ & & 117.9 & - & - & - & - & 116.3 & 114.7 & 114.4 \\
\hline Tyr C $\zeta$ & & 157.6 & - & - & - & - & - & 154.7 & 154.6 \\
\hline Tyr $\mathrm{N}^{\mathrm{H}}$ & & 118.9 & 121.6 & 119.6 & - & - & 118.4 & - & - \\
\hline Gly $\mathrm{H}^{\mathrm{N}}$ & \multirow{5}{*}{6} & 8.34 & 8.35 & 8.28 & - & - & 8.11 & - & 8.4 \\
\hline Gly $\mathrm{H}_{\alpha}$ & & 3.93 & - & - & - & - & $3.72-3.82$ & - & - \\
\hline Gly CO & & 173.9 & 172.1 & 175.4 & 172.7 & 171.0 & $170.1-170.6$ & $170.0-172.3$ & $168.2 / 170.3$ \\
\hline Gly $\mathrm{C} \alpha$ & & 45.4 & 45.1 & 46.9 & 45.0 & 44.2 & 43.4 & $42.2-45.0$ & 41.6 \\
\hline Gly $\mathrm{N}^{\mathrm{H}}$ & & 110.0 & 109.8 & 107.9 & - & - & 106.1 & - & 107.6 \\
\hline Tyr $\mathrm{H}^{\mathrm{N}}$ & \multirow{10}{*}{7} & 8.05 & 8.77 & 8.10 & - & - & 7.91 & - & - \\
\hline Tyr $\mathrm{H}_{\alpha}$ & & 4.61 & - & - & - & - & $4.45-4.51$ & - & - \\
\hline Tyr CO & & 174.9 & 174.3 & 177.1 & - & - & 173.0 & 173.9 & 170.1 \\
\hline Tyr C $\alpha$ & & 57.4 & 56.6 & 60.6 & - & - & 55.8 & 58.4 & 53.0 \\
\hline Tyr $\mathrm{C} \beta$ & & 38.9 & 41.0 & 38.1 & - & - & 37.8 & 34.7 & 38.5 \\
\hline Tyr $\mathrm{C} \gamma$ & & 123.7 & - & - & - & - & - & 126.5 & 126.3 \\
\hline Tyr C $\delta$ & & 133.1 & - & - & - & - & 131.6 & 129.0 & 129.6 \\
\hline Tyr C $\epsilon$ & & 117.9 & - & - & - & - & 116.3 & 114.7 & 114.4 \\
\hline Tyr C $\zeta$ & & 157.6 & - & - & - & - & - & 154.7 & 154.6 \\
\hline Tyr $\mathrm{N}^{\mathrm{H}}$ & & 118.9 & 121.6 & 119.6 & - & - & 118.4 & - & - \\
\hline Gly $\mathrm{H}^{\mathrm{N}}$ & \multirow{5}{*}{8} & 8.34 & 8.35 & 8.28 & - & - & 8.10 & - & 8.4 \\
\hline Gly $\mathrm{H}_{\alpha}$ & & 3.93 & - & - & - & - & $3.72-3.82$ & - & - \\
\hline Gly CO & & 173.9 & 172.1 & 175.4 & 172.7 & 171.0 & 170.6 & $170.0-172.3$ & $168.2 / 170.3$ \\
\hline Gly $\mathrm{C} \alpha$ & & 45.4 & 45.1 & 46.9 & 45.0 & 44.2 & 43.1 & $42.2-45.0$ & 41.6 \\
\hline Gly $\mathrm{N}^{\mathrm{H}}$ & & 110.0 & 109.8 & 107.9 & - & - & 106.1 & - & 107.6 \\
\hline Gly $\mathrm{H}^{\mathrm{N}}$ & \multirow{5}{*}{9} & 8.34 & 8.35 & 8.28 & - & - & 8.12 & - & 8.4 \\
\hline Gly $\mathrm{H}_{\alpha}$ & & 3.93 & - & - & - & - & $3.72-3.82$ & - & - \\
\hline Gly CO & & 173.9 & 172.1 & 175.4 & 172.7 & 171.0 & 170.2 & $170.0-172.3$ & $168.2 / 170.3$ \\
\hline Gly $\mathrm{C} \alpha$ & & 45.4 & 45.1 & 46.9 & 45.0 & 44.2 & 43.4 & $42.2-45.0$ & 41.6 \\
\hline Gly $\mathrm{N}^{\mathrm{H}}$ & & 110.0 & 109.8 & 107.9 & - & - & 105.7 & - & 107.6 \\
\hline Leu $\mathrm{H}^{\mathrm{N}}$ & \multirow{8}{*}{10} & 8.10 & 8.72 & 8.07 & - & - & 7.98 & - & - \\
\hline Leu $\mathrm{H}_{\alpha}$ & & 4.31 & - & - & - & - & $4.30-4.38$ & - & - \\
\hline Leu CO & & 176.4 & 175.4 & 178.2 & - & - & 173.4 & 175.5 & $170.7 / 171.5$ \\
\hline Leu $\mathrm{C} \alpha$ & & 54.7 & 53.8 & 57.4 & - & - & 52.5 & 54.4 & $50.4 / 52.0$ \\
\hline Leu $\mathrm{C} \beta$ & & 42.3 & 44.1 & 41.4 & - & - & 42.1 & 38.3 & $38.8 / 42.5$ \\
\hline Leu $\mathrm{C} \gamma$ & & 26.9 & - & - & - & - & 25.3 & 23.1 & $23.3-23.7$ \\
\hline Leu $\mathrm{C} \delta$ & & 24.5 & - & - & - & - & 24.3 & $18.4 / 21.4$ & 21.5 \\
\hline Leu $\mathrm{N}^{\mathrm{H}}$ & & 122.0 & 124.7 & 120.0 & - & - & 118.0 & - & - \\
\hline Tyr $\mathrm{H}^{\mathrm{N}}$ & \multirow{8}{*}{11} & 8.05 & 8.77 & 8.10 & - & - & 7.95 & - & - \\
\hline Tyr $\mathrm{H}_{\alpha}$ & & 4.61 & - & - & - & - & $4.45-4.51$ & - & - \\
\hline Tyr CO & & 174.9 & 174.3 & 177.1 & - & - & 172.7 & 173.9 & 170.1 \\
\hline Tyr C $\alpha$ & & 57.4 & 56.6 & 60.6 & - & - & 55.7 & 58.4 & 53.0 \\
\hline Tyr C $\beta$ & & 38.9 & 41.0 & 38.1 & - & - & 37.8 & 34.7 & 38.5 \\
\hline Tyr $\mathrm{C} \gamma$ & & 123.7 & - & - & - & - & - & 126.5 & 126.3 \\
\hline Tyr C $\delta$ & & 133.1 & - & - & - & - & 131.6 & 129.0 & 129.6 \\
\hline Tyr C $\epsilon$ & & 117.9 & - & - & - & - & 116.3 & 114.7 & 114.4 \\
\hline
\end{tabular}




\begin{tabular}{|c|c|c|c|c|c|c|c|c|c|}
\hline Tyr C $\zeta$ & 11 & 157.6 & - & - & - & - & - & 154.7 & 154.6 \\
\hline Tyr $\mathrm{N}^{\mathrm{H}}$ & 11 & 118.9 & 121.6 & 119.6 & - & - & 116.6 & - & - \\
\hline Gly $\mathrm{H}^{\mathrm{N}}$ & \multirow{5}{*}{12} & 8.34 & 8.35 & 8.28 & - & - & 8.11 & - & 8.4 \\
\hline Gly $\mathrm{H}_{\alpha}$ & & 3.93 & - & - & - & - & $3.72-3.82$ & - & - \\
\hline Gly CO & & 173.9 & 172.1 & 175.4 & 172.7 & 171.0 & $170.1-170.6$ & $170.0-172.3$ & $168.2 / 170.3$ \\
\hline Gly $\mathrm{C} \alpha$ & & 45.4 & 45.1 & 46.9 & 45.0 & 44.2 & 43.4 & $42.2-45.0$ & 41.6 \\
\hline Gly $\mathrm{N}^{\mathrm{H}}$ & & 110.0 & 109.8 & 107.9 & - & - & 106.1 & - & 107.6 \\
\hline Gly $\mathrm{H}^{\mathrm{N}}$ & \multirow{5}{*}{13} & 8.34 & 8.35 & 8.28 & - & - & 8.12 & - & 8.4 \\
\hline Gly $\mathrm{H}_{\alpha}$ & & 3.93 & - & - & - & - & $3.72-3.82$ & - & - \\
\hline Gly CO & & 173.9 & 172.1 & 175.4 & 172.7 & 171.0 & 170.3 & $170.0-172.3$ & $168.2 / 170.3$ \\
\hline Gly $\mathrm{C} \alpha$ & & 45.4 & 45.1 & 46.9 & 45.0 & 44.2 & 43.4 & $42.2-45.0$ & 41.6 \\
\hline Gly $\mathrm{N}^{\mathrm{H}}$ & & 110.0 & 109.8 & 107.9 & - & - & 105.7 & - & 107.6 \\
\hline Leu $\mathrm{H}^{\mathrm{N}}$ & \multirow{8}{*}{14} & 8.10 & 8.72 & 8.07 & - & - & 7.95 & - & - \\
\hline Leu $\mathrm{H}_{\alpha}$ & & 4.31 & - & - & - & - & $4.30-4.38$ & - & - \\
\hline Leu CO & & 176.4 & 175.4 & 178.2 & - & - & 173.5 & 175.5 & $170.7 / 171.5$ \\
\hline Leu $\mathrm{C} \alpha$ & & 54.7 & 53.8 & 57.4 & - & - & 52.7 & 54.4 & $50.4 / 52.0$ \\
\hline Leu $\mathrm{C} \beta$ & & 42.3 & 44.1 & 41.4 & - & - & 41.7 & 38.3 & $38.8 / 42.5$ \\
\hline Leu $\mathrm{C} \gamma$ & & 26.9 & - & - & - & - & 25.3 & 23.1 & $23.3-23.7$ \\
\hline Leu $\mathrm{C} \delta$ & & 24.5 & - & - & - & - & 24.3 & $18.4 / 21.4$ & 21.5 \\
\hline Leu $\mathrm{N}^{\mathrm{H}}$ & & 122.0 & 124.7 & 120.0 & - & - & 118.4 & - & - \\
\hline Tyr $\mathrm{H}^{\mathrm{N}}$ & \multirow{10}{*}{15} & 8.05 & 8.77 & 8.10 & - & - & 7.95 & - & - \\
\hline Tyr $\mathrm{H}_{\alpha}$ & & 4.61 & - & - & - & - & $4.45-4.51$ & - & - \\
\hline Tyr CO & & 174.9 & 174.3 & 177.1 & - & - & 172.7 & 173.9 & 170.1 \\
\hline Tyr C $\alpha$ & & 57.4 & 56.6 & 60.6 & - & - & 55.7 & 58.4 & 53.0 \\
\hline Tyr C $\beta$ & & 38.9 & 41.0 & 38.1 & - & - & 37.8 & 34.7 & 38.5 \\
\hline Tyr $\mathrm{C} \gamma$ & & 123.7 & - & - & - & - & - & 126.5 & 126.3 \\
\hline Tyr C $\delta$ & & 133.1 & - & - & - & - & 131.6 & 129.0 & 129.6 \\
\hline Tyr C $\epsilon$ & & 117.9 & - & - & - & - & 116.3 & 114.7 & 114.4 \\
\hline Tyr C $\zeta$ & & 157.6 & - & - & - & - & - & 154.7 & 154.6 \\
\hline Tyr $\mathrm{N}^{\mathrm{H}}$ & & 118.9 & 121.6 & 119.6 & - & - & 116.6 & - & - \\
\hline Gly $\mathrm{H}^{\mathrm{N}}$ & \multirow{5}{*}{16} & 8.34 & 8.35 & 8.28 & - & - & 8.04 & - & 8.4 \\
\hline Gly $\mathrm{H}_{\alpha}$ & & 3.93 & - & - & - & - & $3.72-3.82$ & - & - \\
\hline Gly CO & & 173.9 & 172.1 & 175.4 & 172.7 & 171.0 & $170.1-170.6$ & $170.0-172.3$ & $168.2 / 170.3$ \\
\hline Gly $\mathrm{C} \alpha$ & & 45.4 & 45.1 & 46.9 & 45.0 & 44.2 & 43.4 & $42.2-45.0$ & 41.6 \\
\hline Gly $\mathrm{N}^{\mathrm{H}}$ & & 110.0 & 109.8 & 107.9 & - & - & 105.5 & - & 107.6 \\
\hline Gly $\mathrm{H}^{\mathrm{N}}$ & \multirow{5}{*}{17} & 8.34 & 8.35 & 8.28 & - & - & 7.99 & - & 8.4 \\
\hline Gly $\mathrm{H}_{\alpha}$ & & 3.93 & - & - & - & - & $3.72-3.82$ & - & - \\
\hline Gly CO & & 173.9 & 172.1 & 175.4 & 172.7 & 171.0 & 170.3 & $170.0-172.3$ & $168.2 / 170.3$ \\
\hline Gly $\mathrm{C} \alpha$ & & 45.4 & 45.1 & 46.9 & 45.0 & 44.2 & 43.4 & $42.2-45.0$ & 41.6 \\
\hline Gly $\mathrm{N}^{\mathrm{H}}$ & & 110.0 & 109.8 & 107.9 & - & - & 105.3 & - & 107.6 \\
\hline Leu $\mathrm{H}^{\mathrm{N}}$ & \multirow{7}{*}{18} & 8.10 & 8.72 & 8.07 & - & - & 7.95 & - & - \\
\hline Leu $\mathrm{H}_{\alpha}$ & & 4.31 & - & - & - & - & $4.30-4.38$ & - & - \\
\hline Leu CO & & 176.4 & 175.4 & 178.2 & - & - & 173.8 & 175.5 & $170.7 / 171.5$ \\
\hline Leu $\mathrm{C} \alpha$ & & 54.7 & 53.8 & 57.4 & - & - & 52.7 & 54.4 & $50.4 / 52.0$ \\
\hline Leu $\mathrm{C} \beta$ & & 42.3 & 44.1 & 41.4 & - & - & 41.7 & 38.3 & $38.8 / 42.5$ \\
\hline Leu $\mathrm{C} \gamma$ & & 26.9 & - & - & - & - & 25.3 & 23.1 & $23.3-23.7$ \\
\hline Leu $\mathrm{C} \delta$ & & 24.5 & - & - & - & - & 24.3 & $18.4 / 21.4$ & 21.5 \\
\hline
\end{tabular}




\begin{tabular}{|c|c|c|c|c|c|c|c|c|c|}
\hline Leu $\mathrm{N}^{\mathrm{H}}$ & 18 & 122.0 & 124.7 & 120.0 & - & - & 118.4 & - & - \\
\hline Gly $\mathrm{H}^{\mathrm{N}}$ & \multirow{5}{*}{19} & 8.34 & 8.35 & 8.28 & - & - & 8.10 & - & 8.4 \\
\hline Gly $\mathrm{H}_{\alpha}$ & & 3.93 & - & - & - & - & $3.72-3.82$ & - & - \\
\hline Gly CO & & 173.9 & 172.1 & 175.4 & 172.7 & 171.0 & 170.1 & $170.0-172.3$ & $168.2 / 170.3$ \\
\hline Gly $\mathrm{C} \alpha$ & & 45.4 & 45.1 & 46.9 & 45.0 & 44.2 & 43.1 & $42.2-45.0$ & 41.6 \\
\hline Gly $\mathrm{N}^{\mathrm{H}}$ & & 110.0 & 109.8 & 107.9 & - & - & 105.6 & - & 107.6 \\
\hline Tyr $\mathrm{H}^{\mathrm{N}}$ & \multirow{10}{*}{20} & 8.05 & 8.77 & 8.10 & - & - & 8.02 & - & - \\
\hline Tyr $\mathrm{H}_{\alpha}$ & & 4.61 & - & - & - & - & $4.45-4.51$ & - & - \\
\hline Tyr CO & & 174.9 & 174.3 & 177.1 & - & - & 173.1 & 173.9 & 170.1 \\
\hline Tyr C $\alpha$ & & 57.4 & 56.6 & 60.6 & - & - & 55.8 & 58.4 & 53.0 \\
\hline Tyr C $\beta$ & & 38.9 & 41.0 & 38.1 & - & - & 38.0 & 34.7 & 38.5 \\
\hline Tyr $\mathrm{C} \gamma$ & & 123.7 & - & - & - & - & - & 126.5 & 126.3 \\
\hline Tyr C $\delta$ & & 133.1 & - & - & - & - & 131.6 & 129.0 & 129.6 \\
\hline Tyr C $\epsilon$ & & 117.9 & - & - & - & - & 116.3 & 114.7 & 114.4 \\
\hline Tyr C $\zeta$ & & 157.6 & - & - & - & - & - & 154.7 & 154.6 \\
\hline Tyr $\mathrm{N}^{\mathrm{H}}$ & & 118.9 & 121.6 & 119.6 & - & - & 117.0 & - & - \\
\hline Gly $\mathrm{H}^{\mathrm{N}}$ & \multirow{5}{*}{21} & 8.34 & 8.35 & 8.28 & - & - & 8.29 & - & 8.4 \\
\hline Gly $\mathrm{H}_{\alpha}$ & & 3.93 & - & - & - & - & $3.72-3.82$ & - & - \\
\hline Gly CO & & 173.9 & 172.1 & 175.4 & 172.7 & 171.0 & - & $170.0-172.3$ & $168.2 / 170.3$ \\
\hline Gly $\mathrm{C} \alpha$ & & 45.4 & 45.1 & 46.9 & 45.0 & 44.2 & 43.4 & $42.2-45.0$ & 41.6 \\
\hline Gly $\mathrm{N}^{\mathrm{H}}$ & & 110.0 & 109.8 & 107.9 & - & - & 106.6 & - & 107.6 \\
\hline Pro $\mathrm{H}_{\alpha}$ & \multirow{6}{*}{22} & 4.39 & - & - & - & - & - & - & - \\
\hline Pro CO & & 176.5 & 176.0 & 178.0 & 176.5 & - & 172.8 & 173.1 & 173.9 \\
\hline Pro $\mathrm{C} \alpha$ & & 63.0 & 62.7 & 65.3 & 62.0 & - & 60.5 & 63.4 & 61.3 \\
\hline Pro $\mathrm{C} \beta$ & & 32.0 & 32.1 & 31.6 & 32.2 & - & 30.0 & 28.7 & 29.3 \\
\hline Pro $\mathrm{C} \gamma$ & & 27.3 & - & - & 27.1 & - & 25.1 & 23.8 & 23.7 \\
\hline Pro $\mathrm{C} \delta$ & & 50.3 & - & - & 49.2 & - & 47.0 & 46.1 & 46.3 \\
\hline Ala $\mathrm{H}^{\mathrm{N}}$ & \multirow{6}{*}{23} & 8.19 & 8.60 & 8.10 & - & - & 8.10 & - & - \\
\hline Ala $\mathrm{H}_{\alpha}$ & & 4.25 & - & - & - & - & $4.24 / 4.34$ & - & - \\
\hline Ala CO & & 176.9 & 175.6 & 179.1 & 174.3 & 173.9 & 173.7 & 176.8 & $172.0 / 175.5$ \\
\hline Ala $\mathrm{C} \alpha$ & & 52.4 & 50.9 & 54.7 & 49.9 & 50.6 & 49.5 & 51.7 & $47.8 / 51.8$ \\
\hline Ala $C \beta$ & & 19.4 & 21.7 & 18.5 & 22.6 & $18.3 / 21.3$ & 19.3 & 14.0 & $14.4 / 19.5$ \\
\hline Ala $\mathrm{N}^{\mathrm{H}}$ & & 124.1 & 124.9 & 121.8 & - & - & 119.4 & - & - \\
\hline Ala $\mathrm{H}^{\mathrm{N}}$ & \multirow{6}{*}{24} & 8.19 & 8.60 & 8.10 & - & - & 7.79 & - & - \\
\hline Ala $\mathrm{H}_{\alpha}$ & & 4.25 & - & - & - & - & $4.24 / 4.34$ & - & - \\
\hline Ala CO & & 176.9 & 175.6 & 179.1 & 174.3 & 173.9 & 173.5 & 176.8 & $172.0 / 175.5$ \\
\hline Ala $\mathrm{C} \alpha$ & & 52.4 & 50.9 & 54.7 & 49.9 & 50.6 & 49.6 & 51.7 & $47.8 / 51.8$ \\
\hline Ala $C \beta$ & & 19.4 & 21.7 & 18.5 & 22.6 & $18.3 / 21.3$ & 19.1 & 14.0 & $14.4 / 19.5$ \\
\hline Ala $\mathrm{N}^{\mathrm{H}}$ & & 124.1 & 124.9 & 121.8 & - & - & 118.3 & - & - \\
\hline Ala $\mathrm{H}^{\mathrm{N}}$ & \multirow{6}{*}{25} & 8.19 & 8.60 & 8.10 & - & - & 7.93 & - & - \\
\hline Ala $\mathrm{H}_{\alpha}$ & & 4.25 & - & - & - & - & $4.24 / 4.34$ & - & - \\
\hline Ala CO & & 176.9 & 175.6 & 179.1 & 174.3 & 173.9 & 173.8 & 176.8 & $172.0 / 175.5$ \\
\hline Ala $\mathrm{C} \alpha$ & & 52.4 & 50.9 & 54.7 & 49.9 & 50.6 & 49.5 & 51.7 & $47.8 / 51.8$ \\
\hline Ala $C \beta$ & & 19.4 & 21.7 & 18.5 & 22.6 & $18.3 / 21.3$ & 19.3 & 14.0 & $14.4 / 19.5$ \\
\hline Ala $\mathrm{N}^{\mathrm{H}}$ & & 124.1 & 124.9 & 121.8 & - & - & 118.6 & - & - \\
\hline Ser $H^{N}$ & \multirow{2}{*}{26} & 8.28 & 8.55 & 8.14 & - & - & 7.95 & - & - \\
\hline Ser $\mathrm{H}_{\alpha}$ & & 4.48 & - & - & - & - & $4.29 / 4.36$ & - & - \\
\hline
\end{tabular}




\begin{tabular}{|c|c|c|c|c|c|c|c|c|c|}
\hline Ser CO & & 174.4 & 173.5 & 176.0 & - & 173.9 & 171.5 & 173.1 & 173.9 \\
\hline $\operatorname{Ser} \mathrm{C} \alpha$ & \multirow{3}{*}{26} & 58.2 & 57.1 & 61.0 & 57.2 & 56.4 & 56.3 & 59.0 & 53.7 \\
\hline Ser $C \beta$ & & 63.5 & 64.8 & 62.5 & 63.3 & 65.6 & 62.9 & 59.3 & 62.8 \\
\hline Ser $\mathrm{N}^{\mathrm{H}}$ & & 116.3 & 117.4 & 115.1 & - & - & 112.2 & - & - \\
\hline Val $\mathrm{H}^{\mathrm{N}}$ & \multirow{7}{*}{27} & 8.06 & 8.70 & 8.00 & - & - & 7.63 & - & - \\
\hline Val $H_{\alpha}$ & & 4.18 & - & - & - & - & 4.25 & - & - \\
\hline Val CO & & 175.4 & 174.6 & 177.4 & - & - & 172.4 & 175.0 & $171.2 / 172.0$ \\
\hline Val C $\alpha$ & & 61.7 & 60.7 & 65.8 & - & - & 58.7 & 61.8 & $57.5 / 59.1$ \\
\hline Val C $\beta$ & & 32.8 & 33.9 & 31.5 & - & - & 31.9 & 27.9 & $28.9 / 31.5$ \\
\hline Val $\mathrm{C} \gamma$ & & 21.5 & - & - & - & - & 20.5 & 17.5 & $17.3 / 17.7$ \\
\hline Val $\mathrm{N}^{\mathrm{H}}$ & & 120.5 & 122.6 & 119.5 & - & - & 114.6 & - & - \\
\hline Ser $\mathrm{H}^{\mathrm{N}}$ & \multirow{6}{*}{28} & 8.28 & 8.55 & 8.14 & - & - & 8.09 & - & - \\
\hline Ser $\mathrm{H}_{\alpha}$ & & 4.48 & - & - & - & - & $4.29 / 4.36$ & - & - \\
\hline Ser CO & & 174.4 & 173.5 & 176.0 & - & 173.9 & 171.9 & 173.1 & 173.9 \\
\hline Ser $C \alpha$ & & 58.2 & 57.1 & 61.0 & 57.2 & 56.4 & 56.3 & 59.0 & 53.7 \\
\hline Ser $C \beta$ & & 63.5 & 64.8 & 62.5 & 63.3 & 65.6 & 62.9 & 59.3 & 62.8 \\
\hline Ser $\mathrm{N}^{\mathrm{H}}$ & & 116.3 & 117.4 & 115.1 & - & - & 115.6 & - & - \\
\hline Thr $\mathrm{H}^{\mathrm{N}}$ & \multirow{7}{*}{29} & 8.20 & 8.58 & 8.04 & - & - & $7.76 / 7.81$ & - & - \\
\hline Thr $\mathrm{H}_{\alpha}$ & & 4.46 & - & - & - & - & 4.29 & - & - \\
\hline Thr CO & & 174.5 & 173.6 & 176.0 & - & - & 171.6 & 174.4 & 171.1 \\
\hline Thr C $\alpha$ & & 61.4 & 61.0 & 65.5 & - & - & 59.6 & 63.2 & 58.3 \\
\hline Thr C $\beta$ & & 69.4 & 70.4 & 68.1 & - & - & 67.6 & 66.2 & $66.5-68.4$ \\
\hline Thr $\mathrm{C} \gamma$ & & 21.6 & - & - & - & - & 20.8 & 18.0 & 18.3 \\
\hline Thr $\mathrm{N}^{\mathrm{H}}$ & & 114.3 & 117.6 & 114.7 & - & - & 112.1 & - & - \\
\hline Val $\mathrm{H}^{\mathrm{N}}$ & \multirow{7}{*}{30} & 8.06 & 8.70 & 8.00 & - & - & 7.65 & - & - \\
\hline Val $\mathrm{H}_{\alpha}$ & & 4.18 & - & - & - & - & 4.25 & - & - \\
\hline Val CO & & 175.4 & 174.6 & 177.4 & - & - & 172.6 & 175.0 & $171.2 / 172.0$ \\
\hline Val C $\alpha$ & & 61.7 & 60.7 & 65.8 & - & - & 59.3 & 61.8 & $57.5 / 59.1$ \\
\hline Val $\mathrm{C} \beta$ & & 32.8 & 33.9 & 31.5 & - & - & 31.3 & 27.9 & $28.9 / 31.5$ \\
\hline Val $\mathrm{C} \gamma$ & & 21.5 & - & - & - & - & 20.5 & 17.5 & $17.3 / 17.7$ \\
\hline Val $\mathrm{N}^{\mathrm{H}}$ & & 120.5 & 122.6 & 119.5 & - & - & 115.6 & - & - \\
\hline His $\mathrm{H}^{\mathrm{N}}$ & \multirow{8}{*}{31} & 8.22 & 8.70 & 8.00 & - & - & $7.95 / 8.27$ & - & - \\
\hline His $\mathrm{H}_{\alpha}$ & & 4.60 & - & - & - & - & - & - & - \\
\hline $\mathrm{His} \mathrm{CO}$ & & 174.8 & 174.0 & 176.7 & - & - & 171.3 & 173.4 & 171.0 \\
\hline His $\mathrm{C} \alpha$ & & 55.9 & 55.1 & 58.6 & - & - & 52.9 & 53.5 & 51.5 \\
\hline His $\mathrm{C} \beta$ & & 30.2 & 32.2 & 30.0 & - & - & $28.0 / 30.2$ & 27.4 & 29.3 \\
\hline His $\mathrm{C} \delta$ & & 119.7 & - & - & - & - & 115.1 & 115.5 & 114.4 \\
\hline His $\mathrm{C} \epsilon$ & & 137.4 & - & - & - & - & 134.8 & - & - \\
\hline $\operatorname{His} \mathrm{N}^{\mathrm{H}}$ & & 119.7 & 121.9 & 118.0 & - & - & $118.8 / 119.7$ & - & - \\
\hline His $\mathrm{H}^{\mathrm{N}}$ & \multirow{8}{*}{32} & 8.22 & 8.70 & 8.00 & - & - & $8.10 / 8.31$ & - & - \\
\hline His $\mathrm{H}_{\alpha}$ & & 4.60 & - & - & - & - & - & - & - \\
\hline His $\mathrm{CO}$ & & 174.8 & 174.0 & 176.7 & - & - & - & 173.4 & 171.0 \\
\hline His $\mathrm{C} \alpha$ & & 55.9 & 55.1 & 58.6 & - & - & $51.3 / 51.9$ & 53.5 & 51.5 \\
\hline His $\mathrm{C} \beta$ & & 30.2 & 32.2 & 30.0 & - & - & $27.3 / 29.2$ & 27.4 & 29.3 \\
\hline His $\mathrm{C} \delta$ & & 119.7 & - & - & - & - & 115.1 & 115.5 & 114.4 \\
\hline His $\mathrm{C} \epsilon$ & & 137.4 & - & - & - & - & 134.8 & - & - \\
\hline $\operatorname{His} \mathrm{N}^{\mathrm{H}}$ & & 119.7 & 121.9 & 118.0 & - & - & $117.9 / 119.7$ & - & - \\
\hline
\end{tabular}




\begin{tabular}{|c|c|c|c|c|c|c|c|c|c|}
\hline Pro $\mathrm{H}_{\alpha}$ & \multirow{6}{*}{33} & 4.39 & - & - & - & - & - & - & - \\
\hline Pro CO & & 176.5 & 176.0 & 178.0 & 176.5 & - & 173.7 & 173.1 & 173.9 \\
\hline Pro $\mathrm{C} \alpha$ & & 63.0 & 62.7 & 65.3 & 62.0 & - & 60.9 & 63.4 & 61.3 \\
\hline Pro $\mathrm{C} \beta$ & & 32.0 & 32.1 & 31.6 & 32.2 & - & 30.5 & 28.7 & 29.3 \\
\hline Pro $\mathrm{C}_{\gamma}$ & & 27.3 & - & - & 27.1 & - & 25.8 & 23.8 & 23.7 \\
\hline Pro $\mathrm{C} \delta$ & & 50.3 & - & - & 49.2 & - & 47.5 & 46.1 & 46.3 \\
\hline Ser $\mathrm{H}^{\mathrm{N}}$ & \multirow{6}{*}{34} & 8.28 & 8.55 & 8.14 & - & - & 8.38 & - & - \\
\hline Ser $H_{\alpha}$ & & 4.48 & - & - & - & - & $4.29 / 4.36$ & - & - \\
\hline Ser CO & & 174.4 & 173.5 & 176.0 & - & 173.9 & 171.8 & 173.1 & 173.9 \\
\hline $\operatorname{Ser} \mathrm{C} \alpha$ & & 58.2 & 57.1 & 61.0 & 57.2 & 56.4 & 56.8 & 59.0 & 53.7 \\
\hline Ser $C \beta$ & & 63.5 & 64.8 & 62.5 & 63.3 & 65.6 & 62.7 & 59.3 & 62.8 \\
\hline Ser $\mathrm{N}^{\mathrm{H}}$ & & 116.3 & 117.4 & 115.1 & - & - & 114.5 & - & - \\
\hline Thr $\mathrm{H}^{\mathrm{N}}$ & \multirow{7}{*}{35} & 8.20 & 8.58 & 8.04 & - & - & 7.67 & - & - \\
\hline Thr $\mathrm{H}_{\alpha}$ & & 4.46 & - & - & - & - & 4.29 & - & - \\
\hline Thr CO & & 174.5 & 173.6 & 176.0 & - & - & 171.9 & 174.4 & 171.1 \\
\hline Thr C $\alpha$ & & 61.4 & 61.0 & 65.5 & - & - & 59.7 & 63.2 & 58.3 \\
\hline Thr C $\beta$ & & 69.4 & 70.4 & 68.1 & - & - & 67.7 & 66.2 & $66.5-68.4$ \\
\hline Thr $\mathrm{C} \gamma$ & & 21.6 & - & - & - & - & 20.8 & 18.0 & 18.3 \\
\hline Thr $\mathrm{N}^{\mathrm{H}}$ & & 114.3 & 117.6 & 114.7 & - & - & 111.1 & - & - \\
\hline
\end{tabular}

\section{References}

1. Liu, J., Cao, D. \& Zhang, L. Molecular dynamics study on nanoparticle diffusion in polymer melts: A test of the stokes-einstein law. J. Phys. Chem. C 112, 6653-6661 (2008).

2. Marsh, J. A. \& Forman-Kay, J. D. Sequence Determinants of Compaction in Intrinsically Disordered Proteins. Biophys. J. 98, 2383-2390 (2010).

3. Rule, G. S. \& Hitchens, T. K. Fundamentals of protein NMR spectrosopy (Springer, Dordrecht, The Netherlands, 2006).

4. Fritzsching, K. J., Hong, M. \& Schmidt-Rohr, K. Conformationally selective multidimensional chemical shift ranges in proteins from a PACSY database purged using intrinsic quality criteria. J. Biomol. NMR 64, 115-130 (2016).

5. Jenkins, J. E. et al. Solid-state NMR evidence for elastin-like $\beta$-turn structure in spider dragline silk. Chem. Commun. 46, 6714-6716 (2010).

6. Jenkins, J. E., Holland, G. P. \& Yarger, J. L. High resolution magic angle spinning NMR investigation of silk protein structure within major ampullate glands of orb weaving spiders. Soft Matter 8, 1947-1954 (2012).

7. Asakura, T., Sugino, R., Yao, J., Takashima, H. \& Kishore, R. Comparative structure analysis of tyrosine and valine residues in unprocessed silk fibroin (silk I) and in the processed silk fiber (silk II) from Bombyx mori using solid-state 13C,15N, and 2H NMR. Biochemistry 41, 4415-4424 (2002). 